\title{
MSCs-derived exosomes attenuate ischemia-reperfusion brain injury and inhibit microglia apoptosis might via exosomal miR-26a-5p mediated suppression of CDK6
}

\author{
Chang Cheng, Xiuying Chen, Yuhan Wang, Wenchao Cheng, Xuzheng Zuo, Weiju Tang and Wen Huang *(D)
}

\begin{abstract}
Background: This study aimed to explore the role of mesenchymal stromal cells (MSCs)-derived exosomes (MSCsExo) in the cerebral ischemia-reperfusion (I/R) injury.

Methods: Exosomes were isolated from MSCs of adult C57BL/6J mice by the gradient centrifugation method. The expression of miR-26a-5p and CDK6 in MSCs-Exo and mice brain tissues were evaluated by qRT-PCR and western blot. miR-26a-5p mimics and miR-NC were transfected into MSCs, and exosomes were isolated from the MSCs stably expressing miR-26a-5p. Then MSCs-Exo-miR-26a-5p mimics or MSCs-Exo-miR-NC was injected into mice through the tail vein, or added into medium to stimulate BV-2 cells. Cell viability was evaluated by CCK-8 assay. Cell apoptosis was detected by flow cytometry. The apoptosis in brain tissues was evaluated by TUNEL staining assay. Bioinformatics analysis and luciferase reporter assay were performed to determine the binding relationship between miR-26a-5p and CDK6.

Results: miR-26a-5p was downregulated and CDK6 was upregulated in MSCs-Exo of MCAO-mice and OGD-induced MSCs. MSCs-Exo-miR-26a-5p mimics significantly reduced cell apoptosis of OGD-injured BV-2 cells. MSCs-Exo-miR-

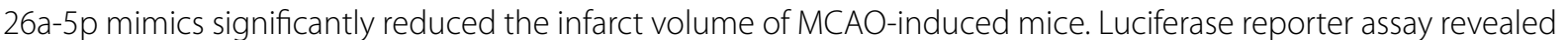
that CDK-6 was a target of miR-26a-5p. In addition, MSCs-Exo-miR-26a-5p mimics significantly decreased the expression of CDK6 in both OGD-induced BV-2 cells and the brain tissues of MCAO-treated mice.

Conclusion: Our results indicated that MSCs-Exo attenuated I/R injury in mice by inhibiting microglia apoptosis might via exosomal miR-26a-5p mediated suppression of CDK6. Our study shed light on the application of MSC-Exo as a potential therapeutic tool for cerebral I/R injury.
\end{abstract}

Keywords: Exosomes, Mesenchymal stromal cells, miR-26a-5p, CDK6, Ischemia-reperfusion injury

\section{Background}

Ischemic stroke is becoming a major leading cause of disability and death worldwide (Barthels and Das 2020). Approximately $85 \%$ of all reported strokes are due to

*Correspondence: WenHuangChongqing@163.com

Department of Neurology, Xinqiao Hospital, Third Military Medical University (Army Medical University), No. 188 Xinqiaozheng Street,

Chongqing 400038, People's Republic of China cerebral ischemia that always occurs when an embolus or thrombus blocks the major cerebral artery and then leads to cell death (Powers 2020). The primary treatments for ischemic stroke are recanalization therapies, which are proved to replenish nutrients and oxygen, and also remove toxic metabolites (Phipps and Cronin 2020). Hence, the identification of new therapies or the original author(s) and the source, provide a link to the Creative Commons licence, and indicate if changes were made. The images or other third party material in this article are included in the article's Creative Commons licence, unless indicated otherwise in a credit line to the material. If material is not included in the article's Creative Commons licence and your intended use is not permitted by statutory regulation or exceeds the permitted use, you will need to obtain permission directly from the copyright holder. To view a copy of this licence, visit http://creativecommons.org/licenses/by/4.0/. 
understanding of critical mediators participated in the progression of cerebral ischemic stroke is still urgent.

Exosomes are nano-scale messengers that carry biomolecular cargo of RNA, DNA, and proteins, and exosomes secreted stem cells have been reported that can regulate various autocrine and paracrine functions to alter cell micro-environment, followed by the progression in various human diseases (Sharma 2018). The potential clinical values of exosomes can be attributed to their characteristics including their surface markers and molecular cargoes (Dorayappan et al. 2016) their ability to cross the brain-blood barrier (BBB) (Kalani et al. 2014), and their potential functions as the mediators of the regenerative responses (Xin 2012). Recently, stem cell therapy has becoming a promising therapeutic option for some neurological disorders including Stroke, Parkinson's disease, Amyotrophic Lateral Sclerosis and Huntington's disease (Yoo et al. 2013). Because the greater benefit on tissue regeneration and repair than the stem cells themselves, stem cell-derived exosomes that are responsible for their therapeutic benefits have begun to be noticed. One of the most popular stem cell-derived exosomes which are widely applied in neurological diseases researches are mesenchymal stromal cells-derived exosomes (MSCs-Exo) (Luarte et al. 2016; Marote et al. 2016). However, the research about MSCs-Exo in cerebral ischemia-reperfusion ( $\mathrm{I} / \mathrm{R})$ injury is lack and attracted us to pay more attention on it.

MicroRNAs (miRNAs) are found in both intracellular and extracellular environments, and also detected in exosomes participated cell-cell communication (Zhang 2015). Increasing reports have revealed a large number of exosome-derived miRNAs in cerebral ischemic stroke. For example, exosomal miR-223 is significantly upregulated in acute ischemic stroke, and high level of exosomal miR-223 is closely associated with the occurrence of acute ischemic stroke and stroke severity (Chen 2017). M2 microglia-derived exosomes has been showed to protect the mouse brain against I/R injury via miR-124 (Song 2019a). Exosomes-derived miR-26a-5p, a newly identified exosomal miRNA, has been revealed to play important roles in human diseases. Human bone MSCsderived exosomes overexpressing miRNA-26a-5p significantly alleviate osteoarthritis development through downregulating PTGS2 (Jin et al. 2020). Dysregulation of exosomal miRNAs such as miR-26a-5p may affect the regulatory pathways associated with clopidogrel-induced liver injury (Freitas 2017). In addition, one previous study demonstrated that miR-26a-5p was downregulated in myocardial $\mathrm{I} / \mathrm{R}$ injury, and overexpression of miR26a-5p effectively improved cell viability and inhibits cell apoptosis in cardiomyocytes upon I/R injury by inhibiting PTEN expression (Xing and Guo 2020). Although exosomal miR-26a-5p played crucial functions in various diseases including I/R injury of some organs, its function and molecular mechanism in cerebral $\mathrm{I} / \mathrm{R}$ injury remain unclear.

CDK6 is known as a classic cell cycle kinase by forming complexes with D-type cyclins, which can phosphorylate the retinoblastoma protein $(\mathrm{Rb})$ to regulate the transition from G1 to S phase (Tigan et al. 2016). Previous studies reported that CDK6 was closely involved in the biological processes including cell proliferation, apoptosis and the transition from $\mathrm{G} 1$ to $\mathrm{S}$ phase in cerebral ischemic stroke (Li 2018; Demyanenko and Uzdensky 2017). Interestingly, Huang et al. found that miR-26a-5p inhibited the growth of breast cancer cells by downregulating the expression of CDK6 (Huang 2019), suggesting that CDK6 might be a direct or indirect downstream gene of miR-26a-5p. Meanwhile, a miRNA profiling and bioinformatics prediction performed by Canturk et al. indicated that the CDK6 was predicted to be a target of miR-26a-5p, and the axis was significantly associated with the progression of bladder cancer (Canturk 2014), further indicating that CDK6 might be a direct target of miR-26a-5p.

In the present study, we explored the role of exosomal miR-26a-5p/CDK6 axis in cerebral I/R injury. Taken together, our study demonstrated that MSCs-derived exosomes overexpressing miR-26a-5p effectively reduced cell apoptosis of OGD-treated microglia cell line BV-2 cells in vitro, and also attenuated MCAO-induced infarct volume in mice, suggesting that exosomal miR-26a-5p might be a potential therapeutic target for cerebral ischemic stroke.

\section{Methods}

\section{Isolation of MSCs-derived exosomes}

Mesenchymal stem cells (MSCs) were isolated from six adult C57BL/6J mice by the whole bone marrow adherence method as previously described (Maria 2016). $2 \times 10^{6}$ MSCs were cultured in $100 \mathrm{~mm}$ dishes, MSCs were washed with $\mathrm{PBS}$ and kept in fetal bovine serum (FBS)-free L-DMEM medium (Gibco, CA, USA) for $48 \mathrm{~h}$ when cells reached nearly $80-90 \%$ confluence. Then the supernatant was collected and subject to sequential centrifugation to obtain the exosomes according to a previous study (Théry et al. 2006). The precipitated exosomes were stored at $-80{ }^{\circ} \mathrm{C}$.

\section{Characterization of MSCs-derived exosomes}

For transmission electron microscopy (TEM), the isolated exosomes were fixed with $1 \%$ glutaraldehyde, then a drop of fixed exosomes was spotted onto a formvar/carbon-coated grid and negatively stained with $3 \%$ aqueous phosphotungstic acid for $1 \mathrm{~min}$. Subsequently, MSCsderived exosomes were observed under TEM (Hitachi, 
Tokyo, Japan, SU-8010). For flow cytometry analysis of exosomes, MSCs-derived exosomes were mixed with $3 \mu \mathrm{m}$ aldehyde/sulfate latex beads (Invitrogen, Batch Num: 979383) for $10 \mathrm{~min}$ with continuous rotation. $1 \mathrm{M}$ glycine in PBS containing 2\% BSA was added into the mixture to stop the reaction. Beads coated with exosomes were incubated with antibodies CD63-FITC (Lot: GR320523-9, Abcam), CD81-PE (Cat: MA5-17941, Invitrogen) at $37^{\circ} \mathrm{C}$ for $25 \mathrm{~min}$. Then a flow cytometry (FCM) (BD FACSCalibur) was used to detect the mesenchymal markers. In addition, the detection of exosomal markers was introduced in western blot assay by using corresponding antibodies including CD9, CD63, CD81, HSP70 and Calnexin.

\section{Animal model of cerebral ischemia-reperfusion (I/R)} A total of 30 adult C57BL/6J mice (8 weeks old and $250 \mathrm{~g}$ in weight) were provided from Anima Center of Third Military Medical University (Chongqing, China). All animal producers were approved by the Institutional Animal Care and Use Committee of Anima Center of Third Military Medical University (Chongqing, China). Mice with cerebral ischemia was induced by middle cerebral artery occlusion (MCAO) as described previously with minor modifications (Yang 1994). Reperfusion was performed by withdrawing the suture $1 \mathrm{~h}$ after MCAO. For sham group, mice received the same operation except MCAO procedure. $2 \mathrm{~h}$ after reperfusion, $200 \mu \mathrm{L} /$ mice MSCsexosomes-miR-NC or MSCs-exosomes-miR-26a-5p mimics (RiboBio, Guangzhou, China) were immediately injected through the tail vein. The mice in the control group were given an equal volume of normal saline $(n=6$ in each group). All mice were divided into four groups: Sham group, MCAO group, MCAO + MSCs-exosomemiR-NC, and MCAO+MSCs-exosome-miR-26a-5p mimics group.

\section{Determination of infarct size}

After intraperitoneal injection of 3\% sodium pentobarbital, the brains of mice in different groups were removed, placed in 4\% PFA overnight and then fully dehydrated in $30 \%$ sucrose for 2 days. The brain tissues were then cut into coronal sections with approximately $2 \mathrm{~mm}$ in thickness, and then quickly incubated in $2 \%$ TTC solution (Sigma) at $37{ }^{\circ} \mathrm{C}$ for $15 \mathrm{~min}$. Subsequently, the sections were fixed by $4 \%$ paraformaldehyde and photographed under a $\times 100$ optical microscope (Olympus Corporation). The image analysis software Image J 1.43 (National Institutes of Health) was used to evaluate the relative infarct percentage according to the following equation: infarct size $=100 \% \times($ infarcted volume $/$ total brain volume).

\section{MSCs of OGD model}

MSCs were cultured with in Dulbecco's modified Eagle's medium (DMEM, Gibco, USA) supplemented with $10 \%(\mathrm{v} / \mathrm{v})$ fetal bovine serum (FBS, Biological Industries, USA), $100 \mathrm{U} / \mathrm{mL}$ penicillin and $100 \mu \mathrm{g} / \mathrm{mL}$ streptomycin (Gibco, USA) at $37{ }^{\circ} \mathrm{C}$ under $5 \% \mathrm{CO}_{2}$. To mimics the ischemia condition in vitro, cells were firstly induced by oxygen-glucose deprivation (OGD) using deoxygenated glucose-free DMEM medium in an incubator with $95 \% \mathrm{~N}_{2}, 5 \% \mathrm{CO}^{2}$ for 1,2 and $4 \mathrm{~h}$, then cells were transferred into normal condition for an additional $24 \mathrm{~h}$ for re-oxygenation. Cells treated without OGD were used as the control group.

\section{Cell transfection}

MSCs were transfected with $50 \mathrm{nM}$ miR-26a-5p mimics or miR-NC (RiboBio, Guangzhou, China) by using Lipofectamine $^{\circledR} 2000$ Transfection Reagent (Gibco Life Technologies) according to the manufacturer's instructions. The sequences used in this study as follows: miR-26a-5p mimics: 5'-UUCAAGUAAUCCAGG AUAGGCU-3'; miR-NC: 5'-UUCUCCGAACGUGUC ACGUTT- $3^{\prime}$. When needed, the exosomes were isolated from MSCs transfected with miR-26a-5p mimics or miR-NC and used for the treatment of microglia cell line BV-2 cells.

\section{The treatment of microglia}

To simulate an in vivo environment of MCAO in microglia, BV-2 cells (Bioleaf, Shanghai, China) were induced by OGD/R treatment as similarly as the MSCs of OGD model above, and BV-2 cells received $2 \mathrm{~h}$ of OGD followed by $24 \mathrm{~h}$ of re-oxygenation. Then $200 \mu \mathrm{g} / \mathrm{mL}$ MSCs (transfected with miR-26a-5p mimics or miRNC)-derived exosomes were added into the culture medium to explore the effect of MSCs-Exo on microglia function.

\section{CCK-8 assay}

Cell viability was evaluated by using a Cell Counting Kit-8 (CCK-8, Dojindo Molecular Technologies, Gaithersburg, MD). In brief, MSCs were seeded into 96-well plates overnight. After the treatment, $10 \mu \mathrm{L}$ of CCK- 8 reagent was added to each well at 24, 48, 72 and $96 \mathrm{~h}$ and then incubated for another $4 \mathrm{~h}$. The absorbance at $450 \mathrm{~nm}$ was detected with a microplate reader.

\section{Luciferase reporter assay}

The putative binding sites between miR-26a-5p and 3'-UTR of CDK6 were predicted by starBase (http:// starbase.sysu.edu.cn/). The mutant-type (MUT) of 3'-UTR (CDK6- MUT) and wild type (WT) of 3'-UTR 
(CDK6-WT) were amplified and cloned into the pmirGLO dual luciferase reporter vector (Promega, Madison, WI, USA). Then the luciferase reporter plasmids were co-transfected with miR-26a-5p mimics or miRNC into $293 \mathrm{~T}$ cells by using Lipofectamine ${ }^{\circledR} 2000$ Transfection Reagent. $48 \mathrm{~h}$ after transfection, cells were lysed and the relative luciferase activity was detected by the dual-luciferase reporter gene assay (Promega).

\section{RNA extraction and qRT-PCR analysis}

Total RNA was extracted from cultured cells or mice brains by using Trizol reagent (Invitrogen). Single-strand cDNA was synthesized using a universal cDNA synthesis kit (Qiagen, Hilden, Germany) according to the manufacturer's instructions. The expression of targets was tested with a fast real-time PCR system $(7900 \mathrm{HT}, \mathrm{ABI}$, Foster City, CA) by using a SYBR Green master mix (Qiagen). The relative expression change of targets was analyzed by the $2^{-\Delta \Delta C t}$ method with GAPDH and U6 as the internal references. The primers used as follows: miR-26a-5p: forward: $\quad 5^{\prime}$-GACGGTACCTTGTCCCTGAATGTA ACTCG-3' reverse: 5'-GTTCTCGAGAAAGCAGTC CCAGCCTAAA-3'; U6: forward: 5'-CTCGCTTCGGCA GCACA-3', reverse: 5'-AACGCTTCACGAATTTGC GT-3'; GAPDH forward: 5'-CAAGGTCATCCATGA CAACTTTG-3', reverse: 5'-GTCCACCACCCTGTT GCTGTAG- $3^{\prime}$.

\section{Western blot}

Total protein of mice brains or cultured cells was isolated by using RIPA lysis buffer (Beyotime Institute of Biotechnology). Approximately equal amounts of protein were separated by $10 \%$ SDS-PAGE and transferred onto PVDF membranes (Millipore). After blocking with 5\% skim milk, the membranes were incubated with primary antibodies including CD9, CD63, CD81, HSP70, CDK6, Calnexin, cleaved Caspase3 (c-Caspase3), cleaved PARP, and GAPDH antibodies (diluted into 1:1000; Cell Signaling Technology, USA) overnight at $4{ }^{\circ} \mathrm{C}$. On the next day, the membranes were incubated with HRP-labeled secondary antibody at room temperature for $1 \mathrm{~h}$. After washing with TBS-T, the protein bands were visualized by ECL reagent and relative integrated density values were calculated using Image J software, with GAPDH as the internal reference. was quantified by Image-Pro Plus 6.0 software (Media Cybernetic).

\section{Apoptosis analysis}

Cell apoptosis was analyzed by using a commercialized Annexin V-FITC/PI Apoptosis Detection Kit (YEASEN, Shanghai) according to the manufacturer's protocols. Briefly, BV-2 cells were washed with PBS, and re-suspended in $100 \mu \mathrm{L}$ of binding buffer, and $5 \mu \mathrm{L}$ of Annexin
V-FITC and $10 \mu \mathrm{L}$ of propidium iodide (PI) were gently mixed and added into cell suspension, then incubated in the dark for $15 \mathrm{~min}$. Finally, cell apoptosis was detected by flow cytometry (BD FACS Canto II, USA).

\section{In situ detection of fragmented DNA (TUNEL assay)}

Brains tissues were collected and the apoptosis in vivo was evaluated TUNEL staining kit (YEASEN, Shanghai) as the manufacturer's instructions. TUNEL positive brain cells were counted under a fluorescence microscope. Specially, cell nucleus dyed green were considered to be apoptotic cells, and the rate of apoptosis (\%) was calculated as the percentage of TUNEL positive cell nucleus in 5 random fields for each sample.

\section{Statistical analysis}

All data were presented as mean $\pm \mathrm{SD}$, and each experiment was repeated three times. Statistical analysis was performed by using GraphPad Prism 6.0 software. Groups comparison was performed with two tailed Student's t test (two groups) or one-way analysis of variance followed by a post-hoc test (ANOVA; multiple groups). $\mathrm{P}<0.05$ was considered to be significant.

\section{Results}

\section{Characterization of MSCs-derived exosomes}

To ensure the reliability of subsequent experiments, we firstly detected the characterization of MSCs-derived exosomes. Under the transmission electronic microscope (TEM), MSCs-derived exosomes showed widespread, derangement distribution, and conglobation in some areas, and the vesicles exhibited typical exosome morphology with a dimension varying from 30 to $150 \mathrm{~nm}$ (Fig. 1A). Flow cytometry assay indicated that CD63 and CD81 positive rates were $34.84 \%$ and $44.70 \%$, respectively (Fig. 1B), suggesting that MSCs-exosomes isolated were of certain purity. Meanwhile, the expression of exosomal markers was evaluated by western blot, and the results showed that the exosomal markers including CD9, CD63, CD81 and HSP70 were all expressed in MSCs and MSCsderived exosomes (Fig. 1C). In addition, there was obvious change on the expression of Calnexin in MSCs and MSCs-derived exosomes (Fig. 1C). These results suggested that the isolated exosomes could be used for the subsequent experiments.

\section{miR-26a-5p was downregulated and CDK6 was upregulated in MSCs-derived exosomes of MCAO and OGD model}

Next, we explored the expression of miR-26a-5p and CDK6 in exosomes of cerebral $\mathrm{I} / \mathrm{R}$ model in vitro and in vivo. Compared with sham group, the expression of miR-26a-5p was significantly downregulated both in MSCs-derived 


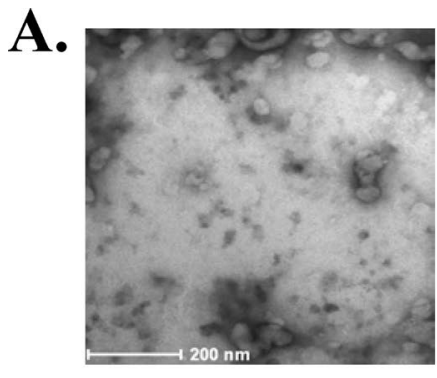

B.
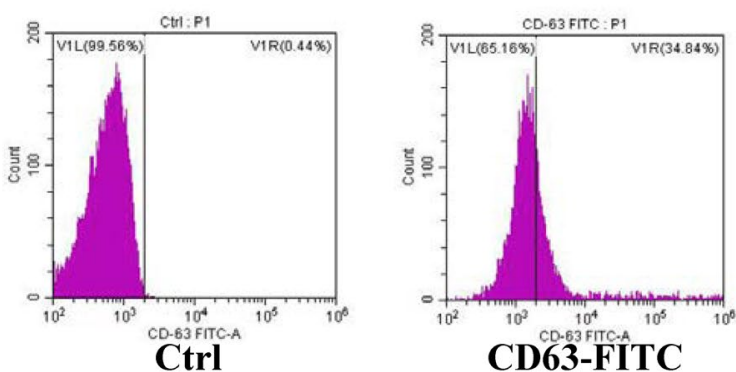

CD63-FITC

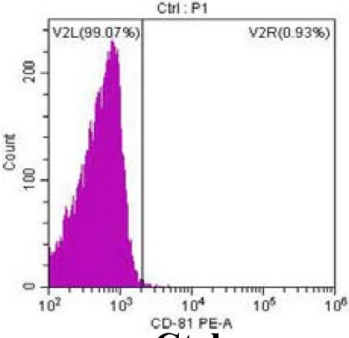

Ctrl

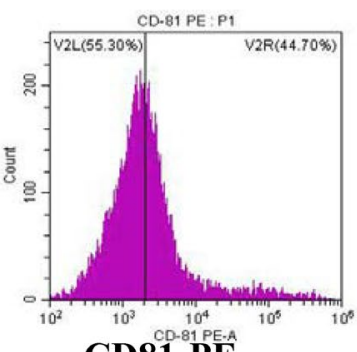

\section{C. mSCs Exosomes}
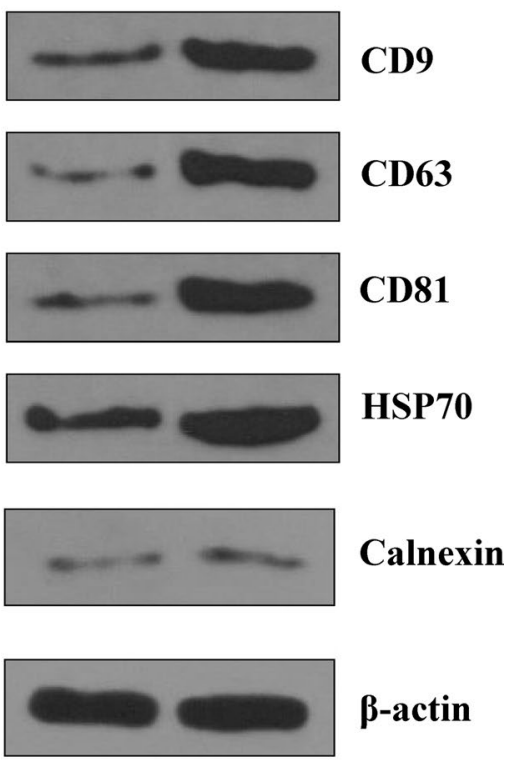

Fig. 1 Characterization of MSCs-derived exosomes. A Morphological change of MSCs-derived exosomes by TEM. B FACS analysis of exosome surface markers CD63 and CD81 in MSCs-derived exosomes. C The protein expression of exosome surface markers (CD9, CD63, CD81, HSP70, Calnexin) in MSCs and MSCs-derived exosomes was evaluated by western blot

exosomes ( $\mathrm{p}<0.01$, Fig. $2 \mathrm{~A})$ and brains tissues $(\mathrm{p}<0.01$, Fig. 2B) of MCAO-induced mice. Similarly, the level of miR-26a-5p in the exosomes of OGD-induced MSCs was also downregulated compared with that in control group (without OGD treatment) ( $\mathrm{p}<0.05$, Fig. $2 \mathrm{C}$ ). In addition, the protein level of CDK6 was increased in MSCs-derived exosomes of MCAO-induced mice compared with that in sham group ( $<<0.01$, Fig. 2D). Meanwhile, the expression of CDK6 was also upregulated in the exosomes of OGD-induced MSCs compared with that in control group $(\mathrm{p}<0.05$, Fig. 2E). These results revealed that miR-26a-5p was downregulated and CDK6 was upregulated in MSCsderived exosomes of MCAO and OGD model.

\section{miR-26a-5p mimics reversed the effects of MSCs-derived exosomes in reducing cell apoptosis of OGD-injured microglia}

To further explore the effect of exosomal miR-26a-5p on microglia function, BV-2 cells were treated by OGD for the given time, and then treated with $200 \mu \mathrm{g} / \mathrm{mL}$ MSCs-Exo-miR-26a-5p mimics or MSCs-Exo-miR-NC. CCK-8 assay indicated that OGD treatment significantly reduced cell viability compared with that in control group ( $\mathrm{p}<0.01$ ), and OGD-treated BV-2 cells cultured with MSCs-Exo-miR-26a-5p showed a greater cell viability than MSC-Exo-miR-NC group ( $\mathrm{p}<0.05$, Fig. 3A). Meanwhile, OGD treatment significantly exacerbated the apoptosis rate of BV-2 cells compared with that in control group $(\mathrm{p}<0.001)$, and the elevated apoptosis of BV-2 cells stimulated by OGD were obviously reduced by MSCs-Exos-miR-26a-5p mimics compared with MSCsExo-miR-NC group $(\mathrm{p}<0.01)$ (Fig. 3B). In addition, the expression of apoptosis-related proteins was evaluated by western blot, and the results showed that the ratio of cleaved-caspase 3/total cleaved caspase- 3 and cleavedPARP/total PARP were increased in BV-2 cells after OGD treatment compared with that in control group $(\mathrm{p}<0.001)$, and the effects were significantly reversed by 

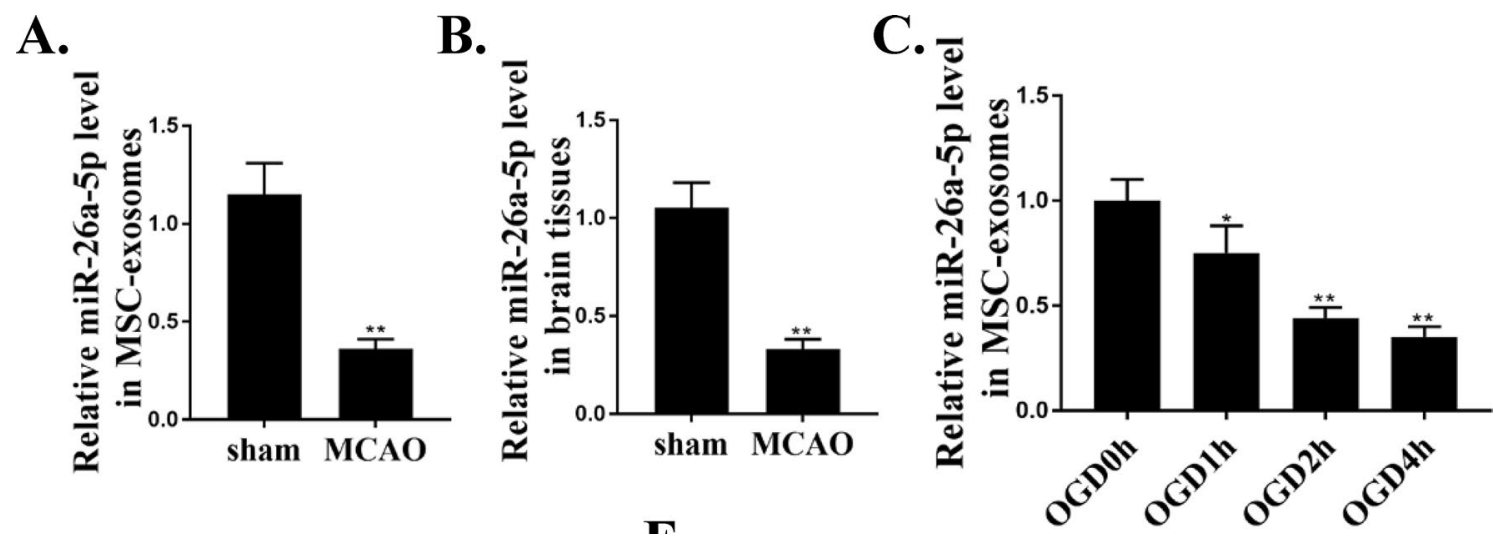

D.

E.
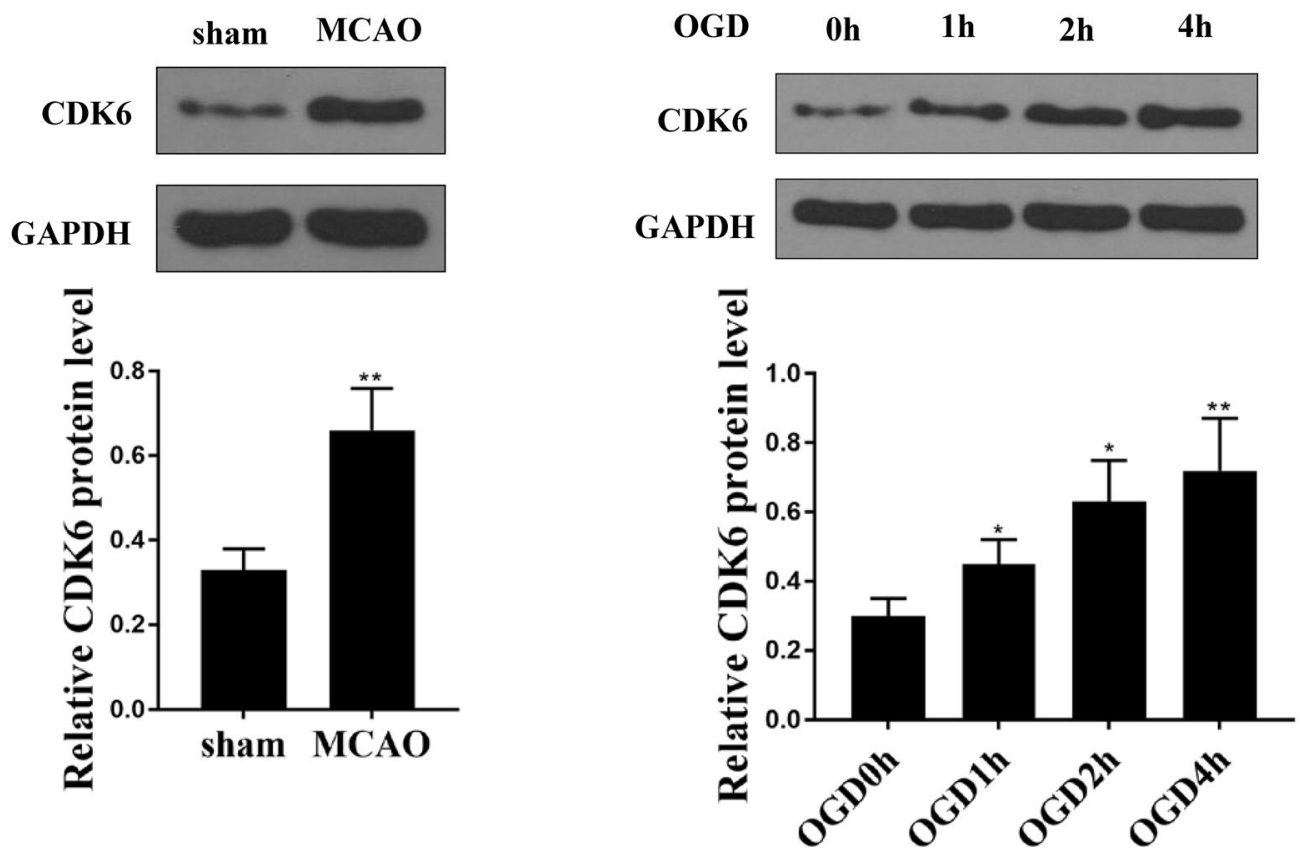

Fig. 2 MiR-26a-5p was downregulated and CDK6 was upregulated in MSCs-derived exosomes of MCAO and OGD model. A and B The mRNA expression of miR-26a-5p in MSCs-derived exosomes $(\mathbf{A})$ and brain tissues (B) of MCAO-induced mice was evaluated by qRT-PCR. CThe level of miR-26a-5p in exosomes of OGD-treated MSCs at indicated time was evaluated by qRT-PCR. D The protein expression of CDK6 in MSCs-derived exosomes of MCAO-induced mice was evaluated by western blot. $\mathbf{E}$ The protein expression of CDK6 in exosomes of OGD-treated MSCS at indicated time was evaluated by western blot. ${ }^{*} p<0.05,{ }^{* *} p<0.01$

MSCs-Exo-miR-26a-5p mimics compared with MSCsExo-miR-NC ( $\mathrm{p}<0.05)$ (Fig. 3C). These results indicated that overexpression of miR-26a-5p could reverse the effects of MSCs-derived exosomes in reducing cell apoptosis of OGD-injured microglia.

\section{MSCs-Exo-miR-26a-5p mimic attenuated ischemia-} reperfusion injury in MCAO/R model

We then explored the neuroprotective effect of MSCs-Exo-miR-26a-5p in vivo through then injection of $200 \mu \mathrm{L} /$ mice MSCs-exosomes-miR-NC or
MSCs-exosomes-miR-26a-5p mimics by the tail vein after $\mathrm{MCAO} / \mathrm{R}$ treatment. The expression of miR-26a-5p in brain tissues was firstly detected by qRT-PCR and the results showed that $\mathrm{MCAO} / \mathrm{R}$ treatment significantly reduced miR-26a-5p level compared with sham operation $(\mathrm{p}<0.001)$, and compared with MSCs-ExomiR-NC group, MSCs-Exo-miR-26a-5p mimics obviously attenuated $\mathrm{MCAO} / \mathrm{R}$-induced downregulation of miR-26a-5p $(\mathrm{p}<0.01)$ (Fig. 4A). The results of TTC staining (Fig. 4B) showed that $\mathrm{MCAO} / \mathrm{R}$ treatment significantly enhanced infarct volume compared with sham 
A.

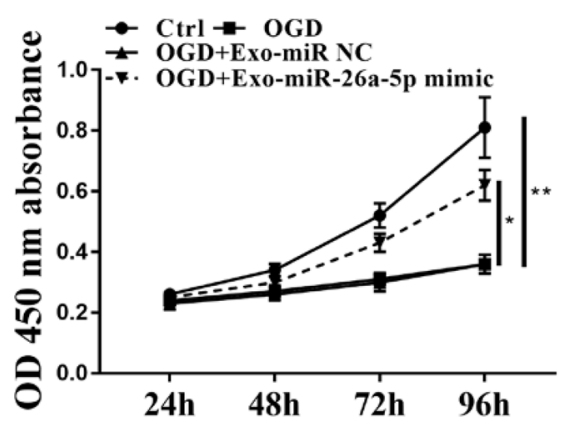

B.

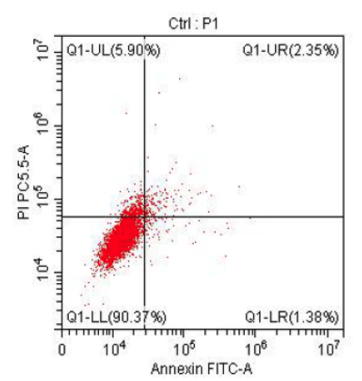

Ctrl

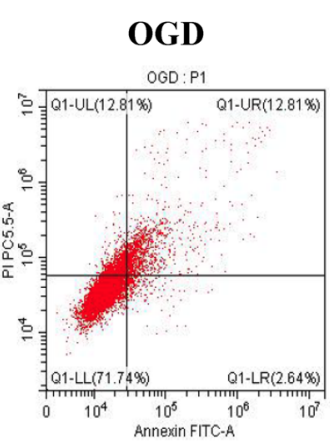

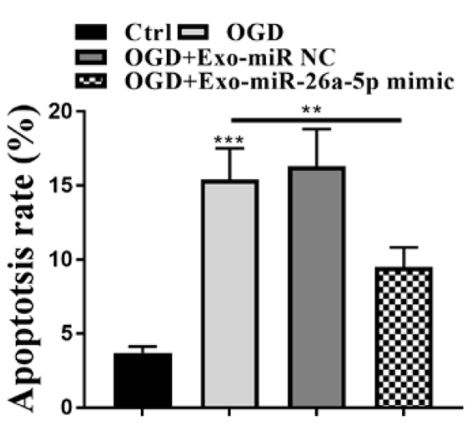

OGD+Exo-miR NC

OGD+Exo-miR-

26a-5p mimic
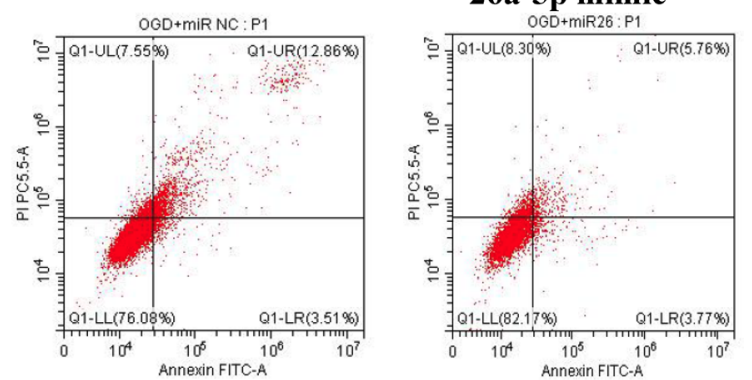

C.
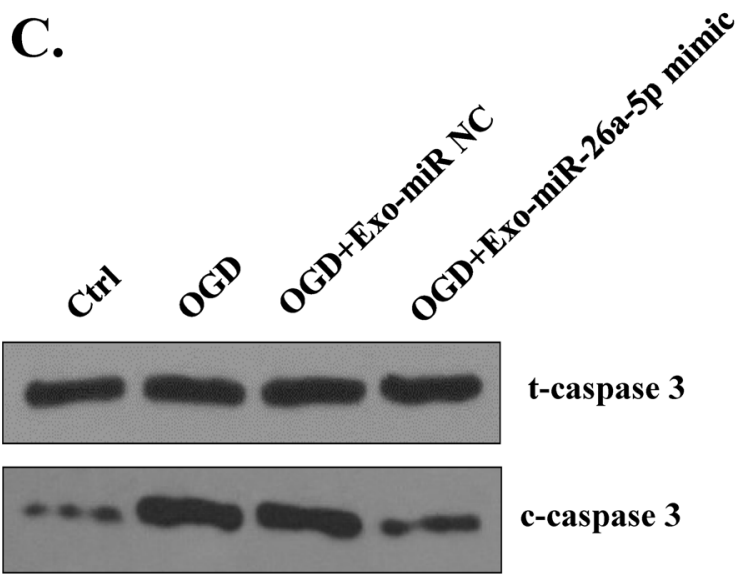

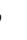

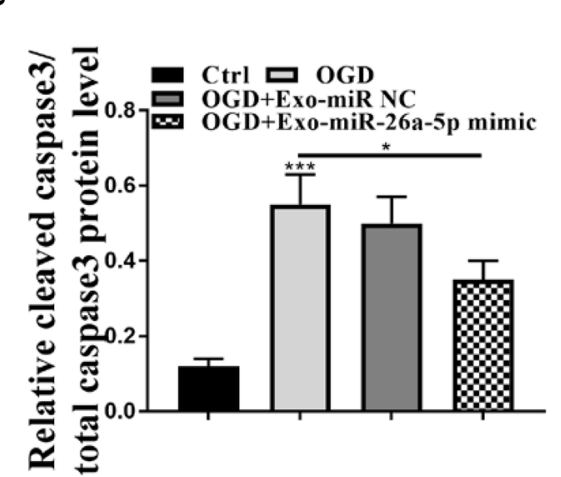

c-caspase 3

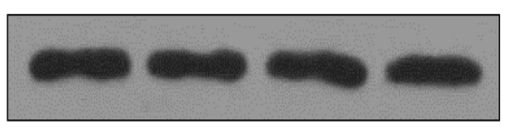

t-PARP

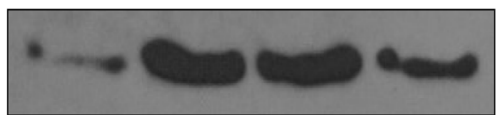

c-PARP

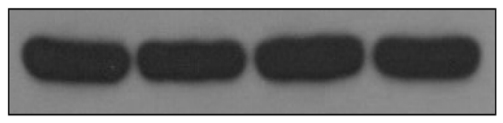

t-caspase 3

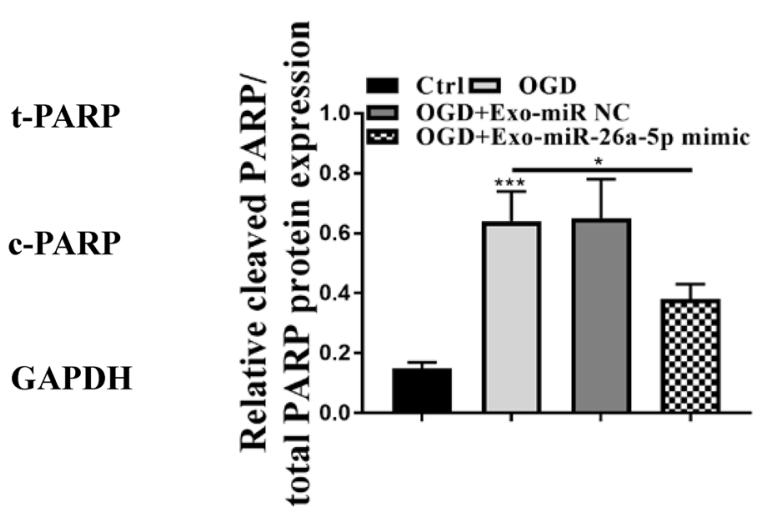

Fig. 3 miR-26a-5p reversed the effects of MSCs-derived exosomes in reducing cell apoptosis of OGD-injured microglia. BV-2 cells were treated by OGD for the given time, and then treated with $200 \mu \mathrm{g} / \mathrm{mL}$ Exo-miR-26a-5p mimics or Exo-miR-NC. A Cell viability was evaluated by CCK-8 assay. B Cell apoptosis was detected by flow cytometry. C The protein expression of apoptosis-related makers (cleaved caspase-3, total caspase-3, cleaved RARP and total RARP) was evaluated by western blot. ${ }^{*} p<0.05,{ }^{* *} p<0.01,{ }^{* * *} p<0.001$ 
(See figure on next page.)

Fig. 4 MSCs-Exo-miR-26a-5p mimic attenuated ischemia-reperfusion injury in MCAO/R model. Mice were treated with MCAO/R, and injected with $200 \mathrm{\mu L} /$ mice MSCs-exosomes-miR-NC or MSCs-exosomes-miR-26a-5p mimics. A The mRNA level of miR-26a-5p in brain tissues was evaluated qRT-PCR. B Representative brain sections of MCAO/R induced mice were detected by TTC staining. CTUNEL staining in brain tissues. D The protein level of apoptosis-related markers (cleaved caspase-3, total caspase-3, cleaved RARP and total RARP) in brain tissues was detected by western blot. ${ }^{*} p<0.05,{ }^{* *} p<0.01,{ }^{* *} p<0.001$

operation $(\mathrm{p}<0.001)$, and compared with Exo-miR-NC group, Exo-miR-26a-5p mimics markedly reduced the infarct percentage $(\mathrm{p}<0.01)$. Meanwhile, cell apoptosis in brain tissues was determined by TUNEL staining and the results indicated that TUNEL positive cells were increased after $\mathrm{MCAO} / \mathrm{R}$ treatment compared with sham operation $(\mathrm{p}<0.001)$, and Exo-miR-26a-5p mimics obviously reduced TUNEL positive cells of mice brains after $\mathrm{MCAO} / \mathrm{R}$ treatment compared with Exo-miR-NC group $(\mathrm{p}<0.01)$ (Fig. 4C). In addition, the expression of apoptosis-related proteins in brain tissues was also evaluated by western blot and the results showed that MCAO/R-treatment significantly increased the ratio of cleaved-caspase 3/total cleaved caspase- 3 and cleaved-PARP/total PARP in brain tissues compared with sham operation $(\mathrm{p}<0.01)$, Exo-miR-26a-5p mimics obviously reduced the ratio of cleaved-caspase 3/total cleaved caspase-3 and cleaved$\mathrm{PARP} /$ total PARP in mice brains induced by $\mathrm{MCAO} / \mathrm{R}$ $(\mathrm{p}<0.05$, Fig. 4D). In addition, Exo-miR-26a-5p mimics exhibited no obvious effect on miR-26a-5p expression, infarct volume, the ration of cleaved-caspase $3 /$ total cleaved caspase- 3 and cleaved-PARP/total PARP in sham-operated mice (Fig. 4A-C). These results suggested that MSCs-Exo-miR-26a-5p mimics attenuated ischemia-reperfusion injury in $\mathrm{MCAO} / \mathrm{R}$ model in vivo.

\section{The effect of miR-26a-5p was partially mediated by CDK6}

To further determine the molecular mechanism of miR$26 a-5 p$, we analyzed the sequence of the $3^{\prime}$-UTR of the human CDK6 gene. We found that there was a putative binding site between miR-26a-5p and 3'-UTR of CDK6 by using Targetscan software (http://www.targetscan. org), suggesting that CDK6 might be a target of mIR26a-5p (Fig. 5A). Then miR-26a-5p mimics or miR-NC was transfected into $293 \mathrm{~T}$ cells and qRT-PCR showed that miR-26a-5p mimics significantly increased the miR26a-5p level compared with miR-NC ( $<<0.01$, Fig. 5B). Meanwhile, luciferase reporter assay was performed in $293 \mathrm{~T}$ cells and the results indicated that miR-26a-5p mimics obviously reduced the relative luciferase activity of CDK6-WT compared with miR-NC $(\mathrm{p}<0.01)$, but had no change on CDK6-MUT (Fig. 5C). Next, miR-26a-5p mimics or miR-NC was transfected into BV-2 cells and we found that miR-26a-5p mimics significantly decreased the expression of CDK6 at both mRNA level $(\mathrm{p}<0.01)$ and protein level $(\mathrm{p}<0.01)$ compared with miR-NC
(Fig. 5D and E). Moreover, we found that OGD/R treatment significantly increased CDK6 level in BV-2 cells compared with control group $(\mathrm{p}<0.01)$, and Exo-miR26a-5p mimics obviously reduced CDK6 level compared with Exo-miR-NC group ( $\mathrm{p}<0.05)$ (Fig. 5F). In addition, $\mathrm{MCAO} / \mathrm{R}$ treatment significantly increased CDK6 level in brain tissues of $\mathrm{MCAO} / \mathrm{R}$ treated mice compared with that in sham operation $(\mathrm{p}<0.01)$, and Exo-miR-26a-5p mimics markedly reduced CDK6 level in mice brains induced by $\mathrm{MCAO} / \mathrm{R}$ treatment compared with ExomiR-NC group $(\mathrm{p}<0.05)$ (Fig. 5G). These data suggested that MSCs-derived exosomes overexpressing miR-26a-5p attenuated cerebral $I / R$ injury might through targeting CDK6.

\section{Discussion}

In this study, we explored the role of exosomal miR$26 a-5 p$ in cerebral I/R injury, and found that miR-26a-5p was downregulated in MSCs-derived exosomes of MCAO and OGD model. In addition, 50, 100 and 200 $\mu \mathrm{L}$ MSCs-Exo-miR-26a-5p were used, and we found that there was no obvious effect on cell viability, apoptosis and infarct volume when 50 and 100 MSCs-Exo-miR-26a-5p were applied. And, $200 \mu \mathrm{L}$ MSCs-Exo-miR-26a-5p mimics effectively reduced cell apoptosis of BV-2 cells submitted to $\mathrm{OGD} / \mathrm{R}$ treatment, and also reduced infarct volume of mice submitted to $\mathrm{MCAO} / \mathrm{R}$ treatment by elevating the expression of CDK6. Our results provided that MSCs-Exo overexpressing miR-26a-5p could effectively attenuate I/R brain injury through targeting CDK6, suggesting that miR-26a-5p might be a novel therapeutic strategy.

A series of miRNAs have been found to be loaded by exosomes in different types of cells, and play crucial roles in neuron injury (Deng 2019). For example, miRNA181a overexpression in MSCs-derived exosomes suppresses inflammatory response after myocardial $\mathrm{I} / \mathrm{R}$ injury (Wei 2019). Cortical neuron-derived exosomal miRNA-181c-3p inhibits neuro-inflammation by downregulating CXCL1 in astrocytes of a rat model with ischemic brain injury (Song 2019b). Exosomes derived from miR-138-5p-overexpressing bone marrow-derived mesenchymal stem cells confers neuroprotection to astrocytes following ischemic stroke via the inhibition of LCN2 (Deng 2019). Exosome-shuttled miR-92b-3p from ischemic preconditioned astrocytes protects neurons 

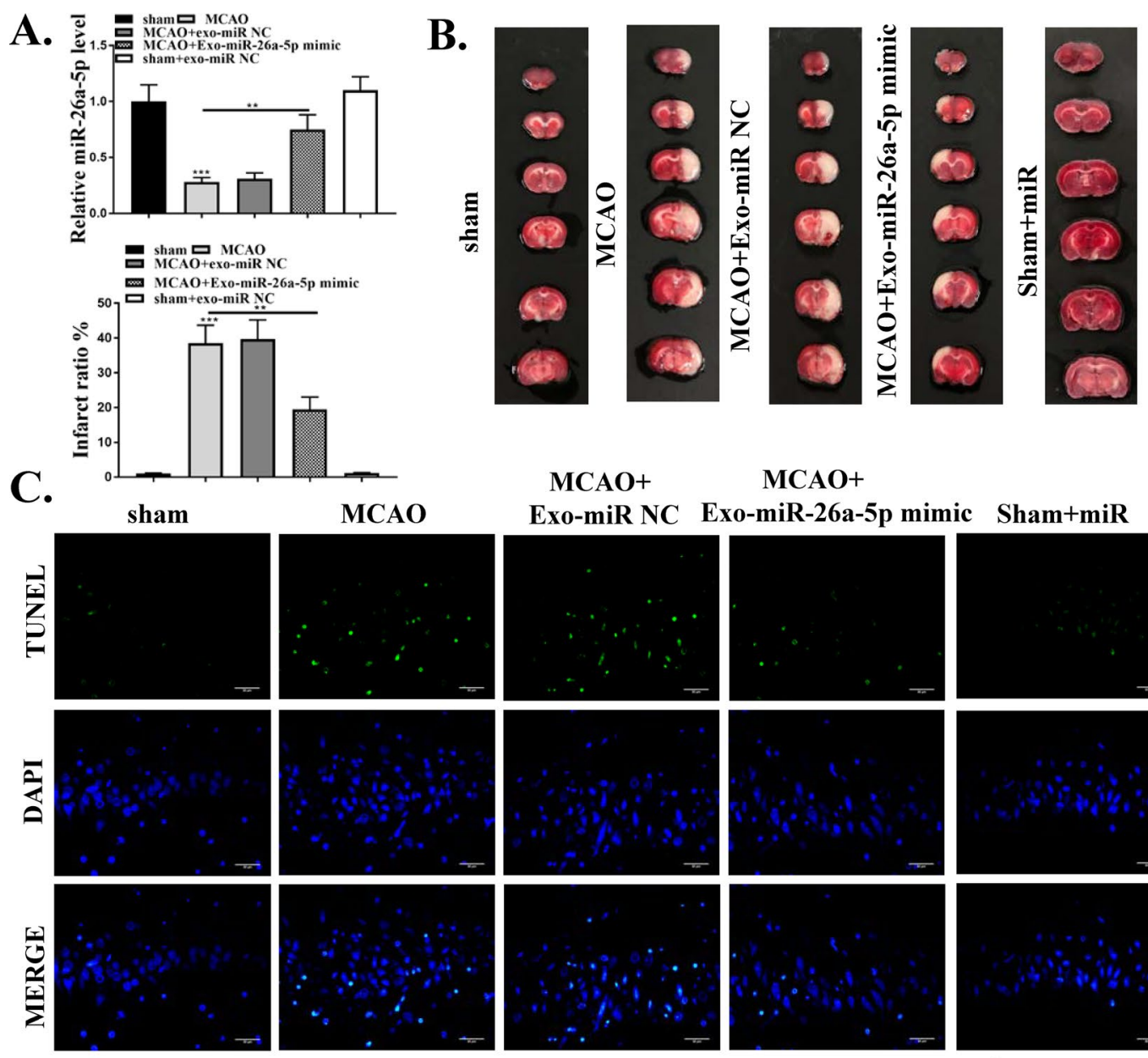

C.
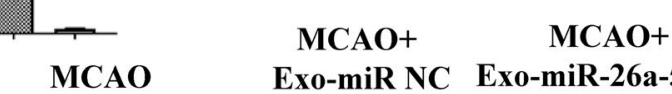

Exo-miR NC Exo-miR-26a-5p mimic Sham+miR
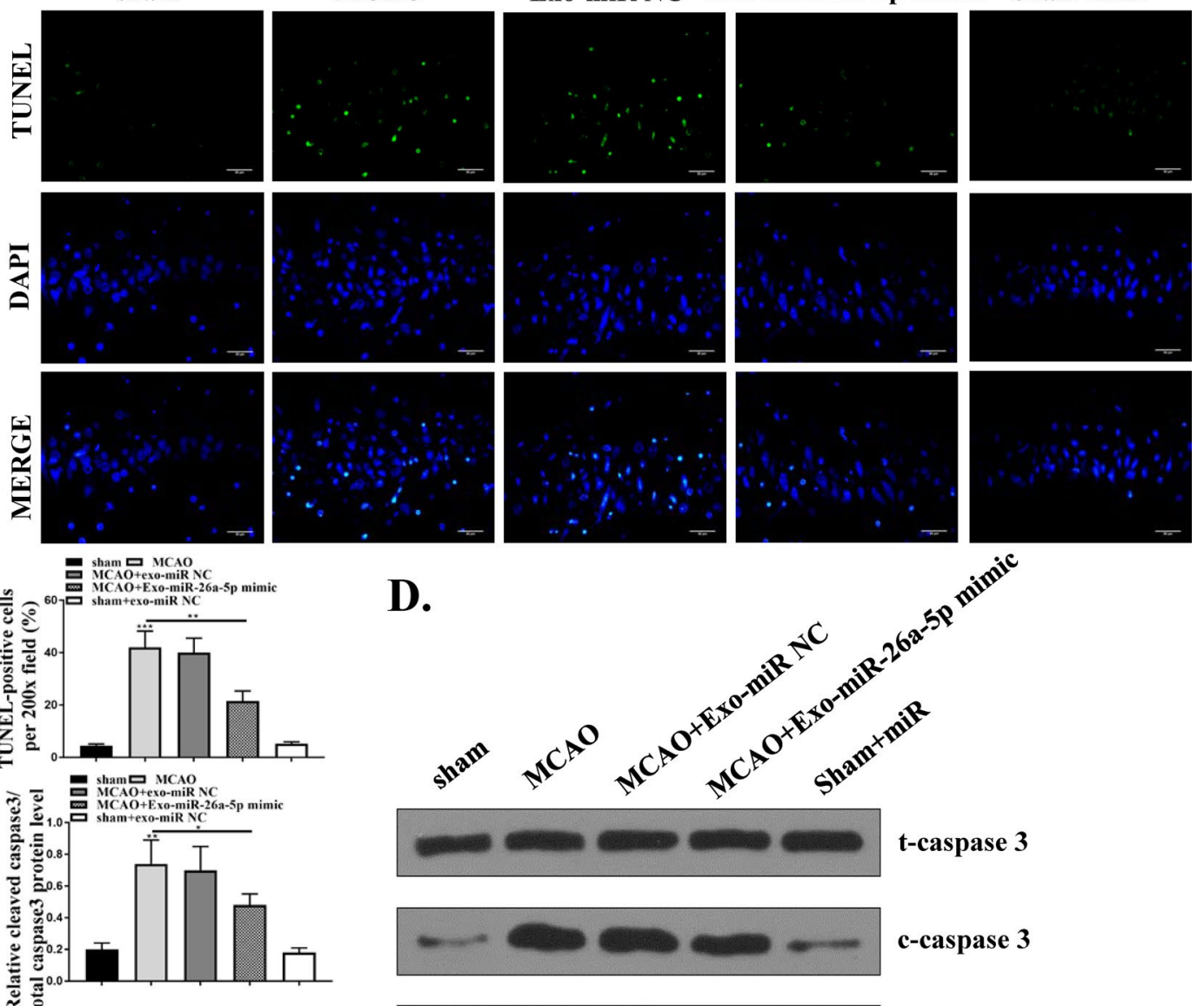

D.
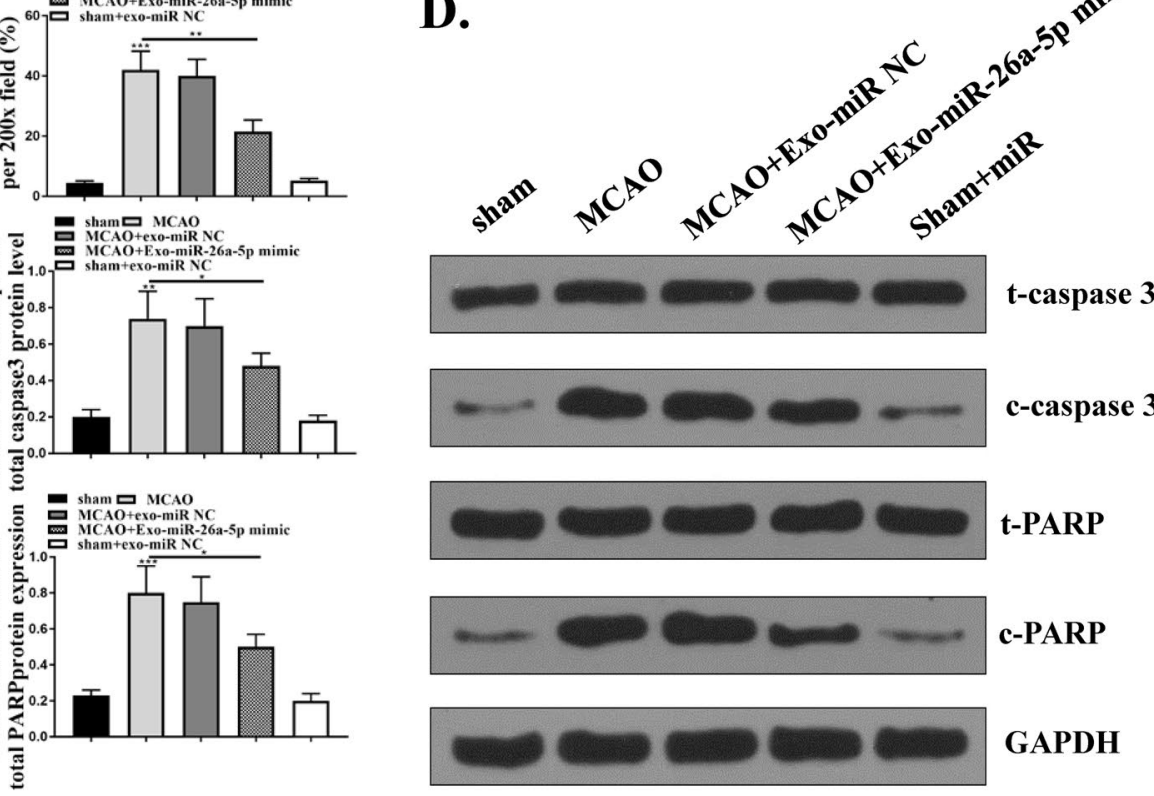
A.

Target: 5' acceguuguacceUUACUGAa 3'

miRNA : 3 ' ucggauaggaccuAAUGAACUu 5'

B.

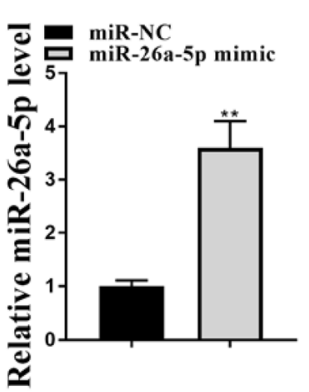

C.

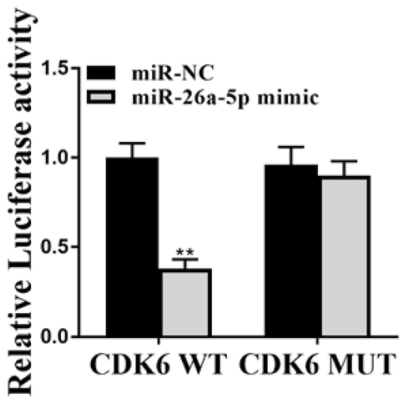

D.
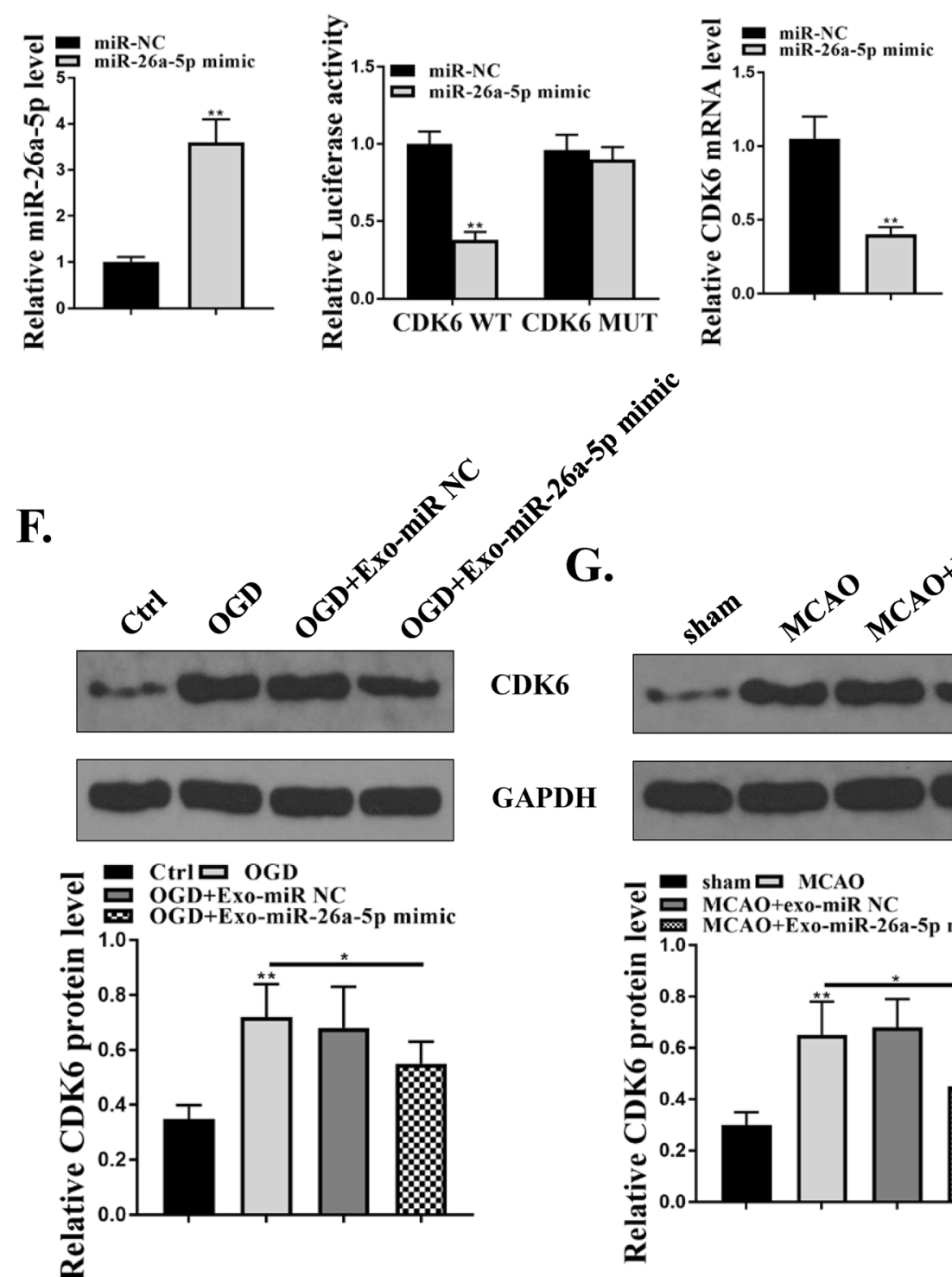

GAPDH
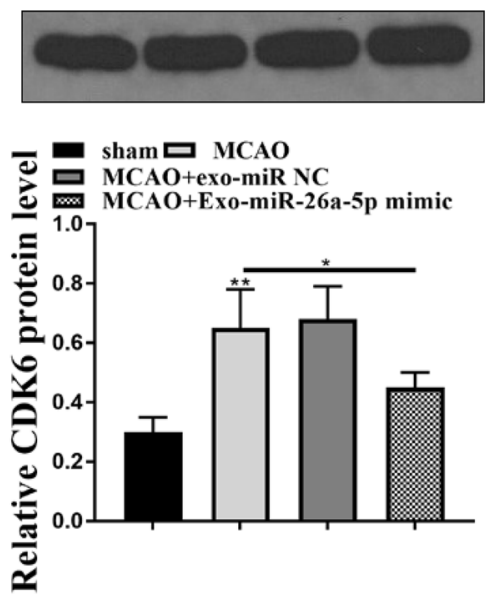

E.
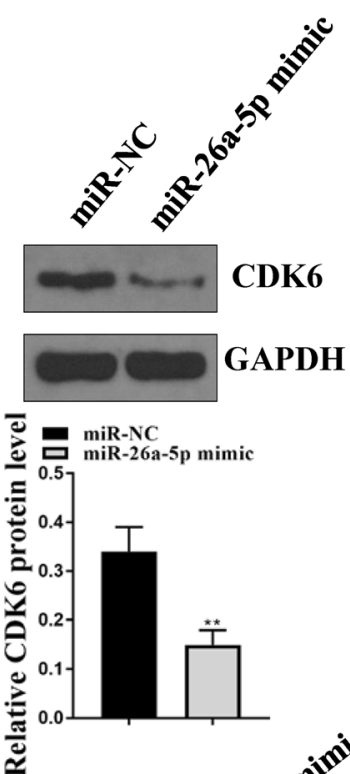

GAPDH

Fig. 5 The effect of miR-26a-5p was partially mediated by CDK6. A The putative binding site between miR-26a-5p and CDK6 was predicted by Targetscan. B 293 T cells were transfected with miR-26a-5p mimics or miR-NC, and the mRNA level of miR-26a-5p was detected by qRT-PCR. C 293 T cells were co-transfected with miR-26a-5p mimics or miR-NC and Luc-CDK6-WT or Luc-CDK6-MUT, and the relative luciferase reporter activity was evaluated by dual luciferase reporter system. $\mathbf{D}$ and $\mathbf{E}$ BV-2 cells were transfected with miR-26a-5p or miR-NC, and the expression of CDK6 was detected by qRT-PCR (D) and western blot (E). F BV-2 cells were treated by OGD/R, and then treated with $200 \mu \mathrm{g} / \mathrm{mL}$ Exo-miR-26a-5p mimics or Exo-miR-NC, and the protein level of CDK6 was evaluated by western blot. G Mice were induced by MCAO/R, and then injected with $200 \mathrm{\mu L} / \mathrm{mice}$ Exo-miR-NC or Exo-miR-26a-5p mimics. The protein level of CDK6 in brain tissues was detected by western blot. ${ }^{*} p<0.05{ }^{* *} p<0.01$ 
against oxygen and glucose deprivation (Xu 2019). Exosomal miR-26a-5p level is decreased in human umbilical cord derived mesenchymal stem cells (hUCMSCs), and exosomal miR-26b-5p from hUCMSCs represses M1 polarization of microglia by targeting $\mathrm{CH} 25 \mathrm{H}$ to inactivate the TLR pathway, then finally relieves nerve injury after cerebral $I / R(L i, 2020)$. In this study, we found that miR-26a-5p was downregulated in MSCs-derived exosomes of MCAO and OGD model. To explore the effect of exosomal miR-26a-5p in cerebral $I / R$ injury, miR-26a-5p overexpressing exosomes were isolated from MSCs and used to stimulate BV-2 cells or injected into $\mathrm{MCAO} / \mathrm{R}$ induced mice. MSCs-Exo-miR-26a-5p mimics effectively reduced cell apoptosis of BV-2 cells submitted to $\mathrm{OGD} / \mathrm{R}$ treatment, and also reduced infarct volume of mice submitted to $\mathrm{MCAO} / \mathrm{R}$ treatment. These results indicated the protective effect of exosomal miR-25a-5p in cerebral I/R injury, which might be regarded as a novel therapeutic target for cerebral ischemic stroke.

Here, we found that CDK6 was upregulated in MSCsderived exosomes of MCAO and OGD model, suggesting the essential role of CDK6 in cerebral I/R injury. Previous studies have reported that CDK6 could serve as a target of miRNAs to participate in neuron injury. MiR-99a overexpression inhibits $\mathrm{H}_{2} \mathrm{O}_{2}$ induced $\mathrm{G1} / \mathrm{S}$ phase transition along with a significant low level of CDK6 in neuro2a cells (Tao 2015). MiR-424 protects from permanent focal cerebral ischemia injury in mice through targeting CDK6 to inhibit microglia activation (Zhao 2013). However, the regulatory network of CDK6 involved in exosomal miRNAs has not been well studied. To explore the specific mechanism of miR-26a-5p in cerebral I/R injury, bioinformatics prediction was performed and suggested that CDK6 might a direct target of exosomal miR-26a-5p. Then luciferase reporter assay further confirmed the correlation between exosome miR-26a-5p and CDK6. Moreover, MSCs-Exo-miR-26a-5p mimics obviously reduced CDK6 level in BV-2 cells after OGD/R treatment, and also reduced CDK6 level in brain tissues of mice induced by $\mathrm{MCAO} / \mathrm{R}$. All these data suggested that the protective effect of MSCs-Exo-miR-26a-5p mimics on cerebral $\mathrm{I} / \mathrm{R}$ injury both in vitro and in vivo might be mediated by CDK6, providing a new highlight of exosomal miRNAs and cell proliferation-related proteins involved in cerebral I/R injury. Our study suggested that MSCs-derived exosomes overexpressing miR-26a-5p might be applied for the personalized treatment against cerebral ischemic stroke.

However, there was a limitation existing in the study: whether high level of CDK6 reversed the protective effect of MSCs-Exo-miR-26a-5p mimics on cerebral I/R injury, which needed to be further investigated in the subsequent experiments.

\section{Conclusion}

In summary, our results demonstrated that MSCs-derived exosomes could effectively attenuate $\mathrm{I} / \mathrm{R}$ injury in vivo and inhibit microglia apoptosis in vitro might through exosomal miR-26a-5p mediated downregulation of CDK6, suggesting that miR-26a-5p might be a novel therapeutic target for cerebral I/R injury.

\section{Acknowledgements \\ Not applicable.}

\section{Authors' contributions}

WH conceived and designed the experiments. CC, XC, YW, WC, XZ and WT performed the experiments. CC analyzed the data and wrote the manuscript. All authors read and approved the final manuscript.

\section{Funding}

This work was supported by the National Natural Science Foundation of China (Grant No. 81571129).

\section{Availability of data and materials}

Any additional information related to this study is available from the author for correspondence upon reasonable request.

\section{Declarations}

Ethics approval and consent to participate Not applicable.

\section{Consent for publication}

All authors give the consent for publication.

\section{Competing interests}

The authors declare that they have no competing interests.

Received: 27 October 2020 Accepted: 1 June 2021

Published online: 02 July 2021

\section{References}

Barthels D, Das H. Current advances in ischemic stroke research and therapies. Biochem Biophys Acta. 2020;1866:165260.

Canturk KM, et al. Investigation of key miRNAs and target genes in bladder cancer using miRNA profiling and bioinformatic tools. Mol Biol Rep. 2014:41:8127-35.

Chen $Y$, et al. Increased circulating exosomal miRNA-223 is associated with acute ischemic stroke. Front Neurol. 2017;8:57.

de Freitas RCC, et al. Modulation of miR-26a-5p and miR-15b-5p exosomal expression associated with clopidogrel-induced hepatotoxicity in HepG2 cells. Front Pharmacol. 2017;8:906

Demyanenko S, Uzdensky A. Profiling of signaling proteins in penumbra after focal photothrombotic infarct in the rat brain cortex. Mol Neurobiol. 2017:54:6839-56

Deng $Y$, et al. Exosomes derived from microRNA-138-5p-overexpressing bone marrow-derived mesenchymal stem cells confer neuroprotection to astrocytes following ischemic stroke via inhibition of LCN2. J Biol Eng. 2019;13:71

Dorayappan KDP, Wallbillich JJ, Cohn DE, Selvendiran K. The biological significance and clinical applications of exosomes in ovarian cancer. Gynecol Oncol. 2016:142:199-205.

Huang ZM, et al. MicroRNA-26a-5p inhibits breast cancer cell growth by suppressing RNF6 expression. Kaohsiung J Med Sci. 2019;35:467-73.

Jin Z, Ren J, Qi S. Human bone mesenchymal stem cells-derived exosomes overexpressing microRNA-26a-5p alleviate osteoarthritis via down-regulation of PTGS2. Int Immunopharmacol. 2020;78:105946.

Kalani A, Tyagi A, Tyagi N. Exosomes: mediators of neurodegeneration, neuroprotection and therapeutics. Mol Neurobiol. 2014;49:590-600. 
Li G, et al. Diagnostic and immunosuppressive potential of elevated mir-424 levels in circulating immune cells of ischemic stroke patients. Aging Dis. 2018:9:172-81.

Li G, et al. Exosomes-carried microRNA-26b-5p regulates microglia M1 polarization after cerebral ischemia/reperfusion. Cell Cycle. 2020;19:1022-35.

Luarte A, Bátiz LF, Wyneken U, Lafourcade C. Potential therapies by stem cellderived exosomes in CNS diseases: focusing on the neurogenic niche. Stem Cells Int. 2016;2016:5736059.

Maria AT, et al. Human adipose mesenchymal stem cells as potent anti-fibrosis therapy for systemic sclerosis. J Autoimmun. 2016;70:31-9.

Marote A, Teixeira FG, Mendes-Pinheiro B, Salgado AJ. MSCs-derived exosomes: cell-secreted nanovesicles with regenerative potential. Front Pharmacol. 2016:7:231.

Phipps MS, Cronin CA. Management of acute ischemic stroke. BMJ. 2020;368:16983.

Powers WJ. Acute ischemic stroke. N Engl J Med. 2020;383:252-60.

Sharma A. Role of stem cell derived exosomes in tumor biology. Int J Cancer. 2018;142:1086-92.

Song Y, et al. M2 microglia-derived exosomes protect the mouse brain from ischemia-reperfusion injury via exosomal miR-124. Theranostics. 2019a;9:2910-23.

Song $\mathrm{H}$, et al. Cortical neuron-derived exosomal microRNA-181c-3p inhibits neuroinflammation by downregulating CXCL1 in astrocytes of a rat model with ischemic brain injury. NeurolmmunoModulation. 2019b;26:217-33.

Tao Z, et al. Neuroprotective effect of microRNA-99a against focal cerebral ischemia-reperfusion injury in mice. J Neurol Sci. 2015;355:113-9.

Théry C, Amigorena S, Raposo G, Clayton A. Isolation and characterization of exosomes from cell culture supernatants and biological fluids. Curr Protoc Cell Biol. 2006;3:22.
Tigan AS, Bellutti F, Kollmann K, Tebb G, SexI V. CDK6-a review of the past and a glimpse into the future: from cell-cycle control to transcriptional regulation. Oncogene. 2016;35:3083-91.

Wei Z, et al. miRNA-181a over-expression in mesenchymal stem cell-derived exosomes influenced inflammatory response after myocardial ischemiareperfusion injury. Life Sci. 2019;232:116632.

Xin $\mathrm{H}$, et al. Exosome-mediated transfer of miR-133b from multipotent mesenchymal stromal cells to neural cells contributes to neurite outgrowth. Stem Cells (dayton, Ohio). 2012;30:1556-64.

Xing X, Guo S. miR-26a-5p protects against myocardial ischemia/reperfusion injury by regulating the PTEN/PI3K/AKT signaling pathway. Braz J Med Biol Res. 2020;53:e9106.

$\mathrm{Xu}$ L, et al. Exosome-shuttled miR-92b-3p from ischemic preconditioned astrocytes protects neurons against oxygen and glucose deprivation. Brain Res. 2019;1717:66-73.

Yang $G$, et al. Human copper-zinc superoxide dismutase transgenic mice are highly resistant to reperfusion injury after focal cerebral ischemia. Stroke. 1994;25:165-70.

Yoo J, Kim HS, Hwang DY. Stem cells as promising therapeutic options for neurological disorders. J Cell Biochem. 2013;114:743-53.

Zhang J, et al. Exosome and exosomal microRNA: trafficking, sorting, and function. Genomics Proteomics Bioinform. 2015;13:17-24

Zhao $\mathrm{H}$, et al. miRNA-424 protects against permanent focal cerebral ischemia injury in mice involving suppressing microglia activation. Stroke. 2013;44:1706-13.

\section{Publisher's Note}

Springer Nature remains neutral with regard to jurisdictional claims in published maps and institutional affiliations.
Ready to submit your research? Choose BMC and benefit from:

- fast, convenient online submission

- thorough peer review by experienced researchers in your field

- rapid publication on acceptance

- support for research data, including large and complex data types

- gold Open Access which fosters wider collaboration and increased citations

- maximum visibility for your research: over 100M website views per year

At BMC, research is always in progress.

Learn more biomedcentral.com/submissions 